\title{
Maternal cohort, time of stillbirth, and maternal age effects in Italian stillbirth mortality
}

\author{
Fabio Parazzini, Carlo La Vecchia, Guerrino Mezzanotte, Luigi Fedele
}

\begin{abstract}
Study objective-The aim of the study was to investigate factors in stillbirth mortality in Italy.

Design-Italian stillbirth data from 1955 to 1979 were analysed using a log-linear Poisson model with arbitrary constraints on the parameters to identify the effects of maternal birth cohort, calendar period of stillbirth and maternal age.
\end{abstract}

Setting-Stillbirth data for the whole of Italy were obtained from the Italian Central Institute of Statistics for the years under examination. During this period the criteria for defining stillbirth did not change and the quality of birth registration was similar.

Main results-Decreases in stillbirth mortality were marked and steady, starting for the generations born since 1920 . A period effect in stillbirth trends was shown, but this was apparently smaller than the cohort trend.

Conclusions-The cohort trend suggests that long term improvements in socioeconomic and general health conditions may be important factors in the diminishing stillbirth rates, as well as short term advances in obstetric care. Alternatively the cohort effect could be attributed, at least in part, to an age-period interaction, since the downward trends were more pronounced in younger women.

Stillbirth rates, in Italy $^{1}$ as in most other countries, ${ }^{2}$ have dropped markedly over the last decades. Socioeconomic and demographic factors and improved medical care have been claimed to be responsible for these trends. ${ }^{3}$

Stillbirth rates are, in principle, influenced by the mother's general health status as well as by medical care in pregnancy. Likewise, maternal health status is determined by her condition in pregnancy, but also by her general health over her whole life. For instance, the secular increase in the height of the population may have had a favourable impact on the pelvic structure and hence on outcome of delivery. Another example of the potential influence of early malnutrition on subsequent reproductive outcome is given by increased stillbirth rate due to malformations of the central system observed in Aberdeen, Scotland, in the cohort of women born in the 1930 s, the calendar period in which the economic depression was at its worst in that area. ${ }^{4}$

Thus, in any analysis of stillbirth rates, a maternal cohort effect (attributable to lifelong maternal health status) and a period effect (determined, for instance, by application in perinatal care of newer more effective treatments or, more generally, of short term advances in pregnancy care and assistance) may interact in a complex way.

The possibility of disentangling, through appropriate statistical models, the effect of maternal cohort of birth from that of calendar period when stillbirth occurred is therefore of potential interest for understanding the role of short and long term social and medical factors as determinants of stillbirth rates. We have thus applied a log-linear age/period/cohort model to Italian stillbirth data between 1955 and 1979.

\section{Methods}

The materials used are those previously described for cross sectional studies on perinatal mortality in Italy. ${ }^{15}$

Briefly, since 1955 numbers of live and stillbirths have been published annually by the Italian Central Institute of Statistics (ISTAT) within strata of maternal age. ${ }^{6}$ During the period considered, the criteria of definition of stillbirth were not changed, and the quality of birth registration was similar (for example, the percentage of missing values of maternal age was less than $1 \%$ during all the period considered).

From the matrices of each five year calendar period (from 1955-59 to 1975-79) and age group (from 15-19 to 45-49 years) the effects of maternal age, maternal cohort of birth, and period of stillbirth were evaluated applying a log-linear Poisson model with arbitrary constraints on the parameters, fitted using the General Linear Interactive Modelling (GLIM) system $^{7}$ with appropriate user supplied routines.

To overcome the major inherent problem of age/period/cohort modelling (ie, the fact that these three factors are not independent, and hence whenever two of them are defined the third is fixed automatically), we first developed the three possible two factor models (age/cohort, age/ period, cohort/period). The age/cohort/period model was subsequently obtained by minimising the sum of the euclidean distances between the three two factor models. Thus the procedure used was conceptually similar, though not equivalent, to that originally described by Osmond and Gardner and applied to cancer mortality ${ }^{8}$ and induced abortion trends in England and Wales. ${ }^{9}$ The procedure of minimisation implemented by the GLIM macros, however, is based on the least squares weighted on the inverse of the loglikelihood of each two factor model, taken as a measure of goodness of fit, ${ }^{10}$ whereas Osmond and Gardner used the mean residual sum of 
squares, minimising the sum of distances as a measure of goodness of fit.

Maternal cohort of birth and period of stillbirth values were averaged to unity. They can therefore be interpreted as the estimated risk of stillbirth for each specific cohort or period compared to the whole of the data considered, while age values are assimilable to rates per 1000 births.

Age and cohort values are always based on five age specific rates from the original matrices, but cohort values related to earlier and more recent periods are based on fewer age specific rates (ie, only one for the 1910 cohort, two for the 1915 cohort and, from the other end, one for the 1960, two for the 1955 cohort and so on). Thus, values for extreme cohorts are clearly subject to greater random variation. Further, the inclusion of any linear component on one factor modifies the parameter estimates for the other factors, ie, there is an inevitable problem of identifiability in any such model. Thus the estimated values should be viewed in relative terms of changes in slopes (ie, second derivatives of the curves), rather than in relation to their absolute values and, in any case, although they may represent a useful summary guide to the interpretation of trends in mortality, the estimates from the model are in no event substitutes for careful examination of age-specific trends. Problems of estimation in age/period/ cohort models are discussed in detail in a separate paper. $^{10}$

\section{Results}

The table gives the matrix age specific rates. Maternal cohorts can be read on the ordinate, age of the mother on the abscissa, and period of
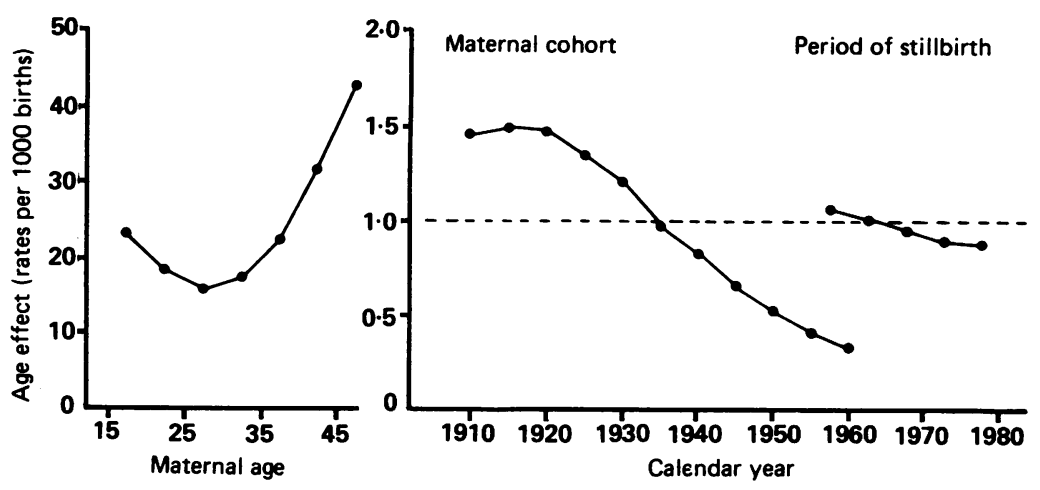

Figure 1 Effect of maternal age, cohort of maternal birth, and period of stillbirth in Italy, 1955-1979.

Italian stillbirth mortality rates/1000 pregnancies, 1955-1979

\begin{tabular}{|c|c|c|c|c|c|c|c|c|}
\hline \multirow{2}{*}{$\begin{array}{l}\text { Period of } \\
\text { stillbirth }\end{array}$} & \multicolumn{7}{|c|}{ Maternal age (years) } & \multirow{2}{*}{$\begin{array}{l}\text { Central year of } \\
\text { maternal } \\
\text { birth cohort }\end{array}$} \\
\hline & $15-19$ & $20-24$ & $25-29$ & $30-34$ & $35-39$ & $40-44$ & $45-49$ & \\
\hline $\begin{array}{r}1955-59 \\
1960-64 \\
1965-69 \\
1970-74 \\
1975-79 \\
\end{array}$ & $\begin{array}{r}19.50 \\
15.29 \\
11.41 \\
8.33 \\
6.90 \\
\end{array}$ & $\begin{array}{r}18 \cdot 71 \\
16 \cdot 18 \\
10 \cdot 97 \\
8 \cdot 39 \\
6.54\end{array}$ & $\begin{array}{r}20 \cdot 53 \\
15.00 \\
12.28 \\
9 \cdot 23 \\
7.65\end{array}$ & $\begin{array}{r}24.95 \\
20.95 \\
16.48 \\
12.27 \\
9.47\end{array}$ & $\begin{array}{l}34 \cdot 89 \\
30 \cdot 39 \\
25 \cdot 26 \\
19 \cdot 93 \\
15 \cdot 42\end{array}$ & $\begin{array}{l}50 \cdot 39 \\
47 \cdot 25 \\
40 \cdot 01 \\
34 \cdot 01 \\
27 \cdot 40\end{array}$ & $\begin{array}{l}66 \cdot 39 \\
64 \cdot 75 \\
59 \cdot 71 \\
49 \cdot 83 \\
46 \cdot 58\end{array}$ & $\begin{array}{l}1910 \\
1915 \\
1920 \\
1925 \\
1930 \\
1935 \\
1940 \\
1945 \\
1950 \\
1955 \\
1960\end{array}$ \\
\hline
\end{tabular}

stillbirth on the diagonal. Stillbirth rates rose with maternal age, and substantially decreased in each subsequent cohort and calendar period.

The results of the age/period/cohort model are plotted in fig 1 . Stillbirth risk increased with maternal age. The excess stillbirths observed in teenagers according to the age/period/cohort model is apparently greater than that observed from direct inspection of cross sectional rates (table, fig 2), in the absence of consideration of the cohort effect. Decreases in stillbirth mortality

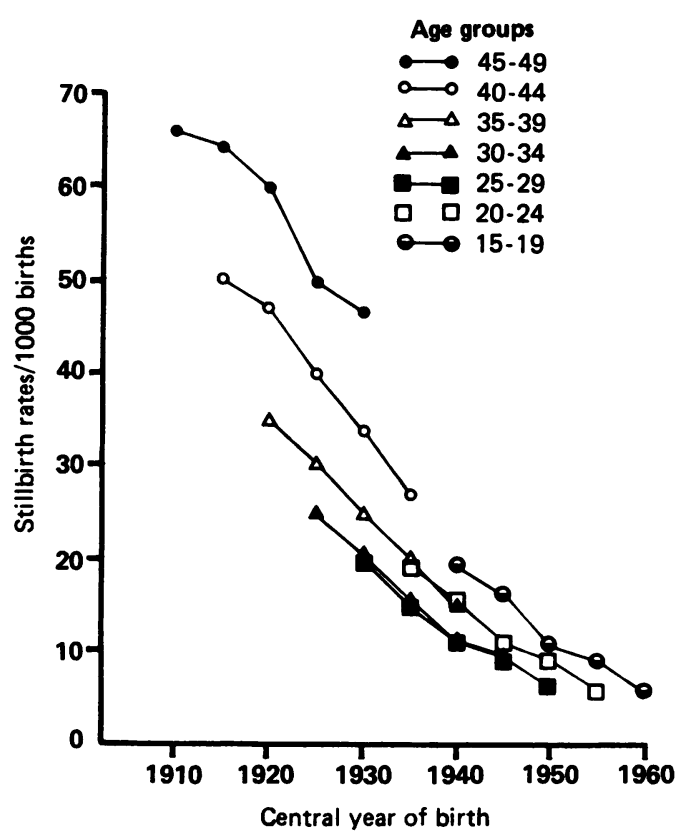

Figure 2 Trend in stillbirth rates in subsequent cohort of maternal birth, Italy, 1955-1979.

were marked and steady for the generations born since 1920. Likewise, a period effect emerged in drops in stillbirth rates; however, this was apparently smaller than the cohort one.

No appreciable differences emerged in period/ cohort/age effect when analysis was conducted according the three broad Italian geographical areas (North, Centre, South; data not shown).

\section{Discussion}

Application of a log-linear Poisson model to Italian stillbirth rates suggested that there was a major cohort component in the decrease, while the declines in the period component were apparently smaller.

The indications given by any such model must be considered with great caution, since there is no way to overcome its non-identifiability property and, therefore, an age-period interaction could spuriously enhance a cohort effect. Further, the importance given by the model to the cohort effect could be explained, at least in part, in terms of limitations of the model, since the number of period parameters estimated was only five as compared with 11 cohort and seven age parameters, and the model tends to weight each effect in direct relation with the corresponding number of parameters. ${ }^{10}$ These findings, nonetheless, merit discussion in epidemiological terms. The model suggests that long term 
improvements in socioeconomic, general health, and obstetric conditions, acting with a generation effect, are a relevant determinant of stillbirth rates, besides short term advances in obstetric care. In this regard socioeconomic status is a well known risk factor for stillbirth. ${ }^{35}$ In Italy, stillbirth rates were, over the period considered, about 2.5 times greater in women with less than five years of education than among those with high school or university degree, and the decrease in stillbirth rates was apparently greater for more educated women (approximately $-5 \%$ per year $v$ $-3 \%$ in the less educated). ${ }^{1}$ The proportion of more educated women (high school or university degree) has increased in more recent Italian generations, representing in the late 1970 s about $20 \%$ of women aged $25-44$ years and $40 \%$ in the 20-25 year age group. Thus, more recent Italian generations are more educated and have better general socioeconomic status and probably healthier lifestyle habits.

In our analysis, cohort effect in stillbirth mortality was marked and steady over the period considered. A rise in stillbirth rates for congenital malformation of the central nervous system on a cohort basis was observed in women born between 1928 and 1934 in Scotland. ${ }^{4}$ In Italy, however, the economic depression of the early 1930 s had a smaller impact on the conditions of the general population, the Italian economy being in that period mostly based on agriculture.

Comparisons of trends within various Italian regions offer further indications that a cohort component plays a role in stillbirth rates. The introduction of new medical treatments, the availability of which is now more uniform in the various Italian regions, particularly following the introduction of a National Health Service in the 1970s, should have provided a rapid reduction to similar values of stillbirth rates, ie, a typical period effect. Stillbirth rates, however, have been decreasing at a rather similar slope in the various regions, leaving relative differences between the regions largely unchanged. ${ }^{511}$ This again suggests a major role for socioeconomic (and hence long term or cohort) factors in Italian stillbirth rates.

This work was conducted within the framework of the CNR (National Research Council, Rome, Italy) Programme on Clinical Pharmacology, "Monitoraggio dell'Uso dei Farmaci" and applied project "Risk factors for disease".

We wish to thank Ms Judy Baggott and Maria Nigro for editorial assistance.

1 Parazzini F, Imazio C, Pampallona S, La Vecchia C. Trends in perinatal neonatal and postneonatal mortality in Italy, 1955-84. Soz Präventivmed 1987; 32: 286-90.

2 United Nations, Department of International Economic and Social Affairs Statistical Office. World statistics in brief. New York: United Nations Organization, 1983.

3 Chamberlain G. Background to perinatal health. Lancet 1979; 2: 1061-3.

4 Baird D. Epidemiology of congenital malformations of the central nervous system in (a) Aberdeen and (b) Scotland. $f$ Biosoc Sci 1974; 6: 113-37.

5 Parazzini F, Negrello I, La Vecchia C. Natimortalità e mortalità nel primo anno di vita nelle diverse regioni Italiane: analisi degli andamenti temporali dal 1955 al 1979. Ann Ostet Ginecol Med Perinat 1986; 107: 5-120.

Ann Ostet Ginecol Med Perinat 1986; 107: 5-120.
6 ISTAT. Annuario di statistiche demografiche. Vols. IVISTAT. Annuario di statistiche demografich
XXVII. Edition 1955-80. Roma, 1958-83.

7 Baker NJ, Nelder JA. The GLIM system: release 3. Oxford: Numerical Algorithms Group, 1978.

8 Osmond C, Gardner MJ, Acheson ED. Analysis of trends in cancer mortality in England and Wales during 1951-80 separating changes associated with period of birth and period of death. $\mathrm{Br} \mathrm{Med} \mathcal{F} 1982 ; 284$ : 1005-8.

9 Ashton JR, Machin D, Osmond C, Balajaran R, Adam SA, Donnan SPB. Trends in induced abortion in England and Wales. $\mathcal{F}$ Epidemiol Community Health 1983; 37: 105-10.

10 Decarli A, La Vecchia C. Age, period and cohort models: Review of knowledge and implementation in GLIM. Rivista Statistica Applicata 1987; 20: 397-410.

11 Parazzini F, La Vecchia C, Negri E. Descriptive epidemiology of perinatal and infant mortality in various Italian geographic areas. Soz Präventivmed 1988; 33: 245-9. 